\title{
Power-Dependent Dual Analyte Tip-Enhanced Raman Spectral Imaging
}

Brian T. O'Callahan, ${ }^{1}$ Ashish Bhattarai, ${ }^{2}$ Zachary D. Schultz, ${ }^{3}$ Patrick Z. El-Khoury, ${ }^{2, *}$

${ }^{1}$ Environmental Molecular Sciences Laboratory, and ${ }^{2}$ Physical Sciences Division, Pacific

Northwest National Laboratory; P.O. Box 999, Richland, WA 99352, USA; ${ }^{3}$ Department of Chemistry and Biochemistry, The Ohio State University, Columbus, OH 43210, USA

*patrick.elkhoury@pnnl.gov 

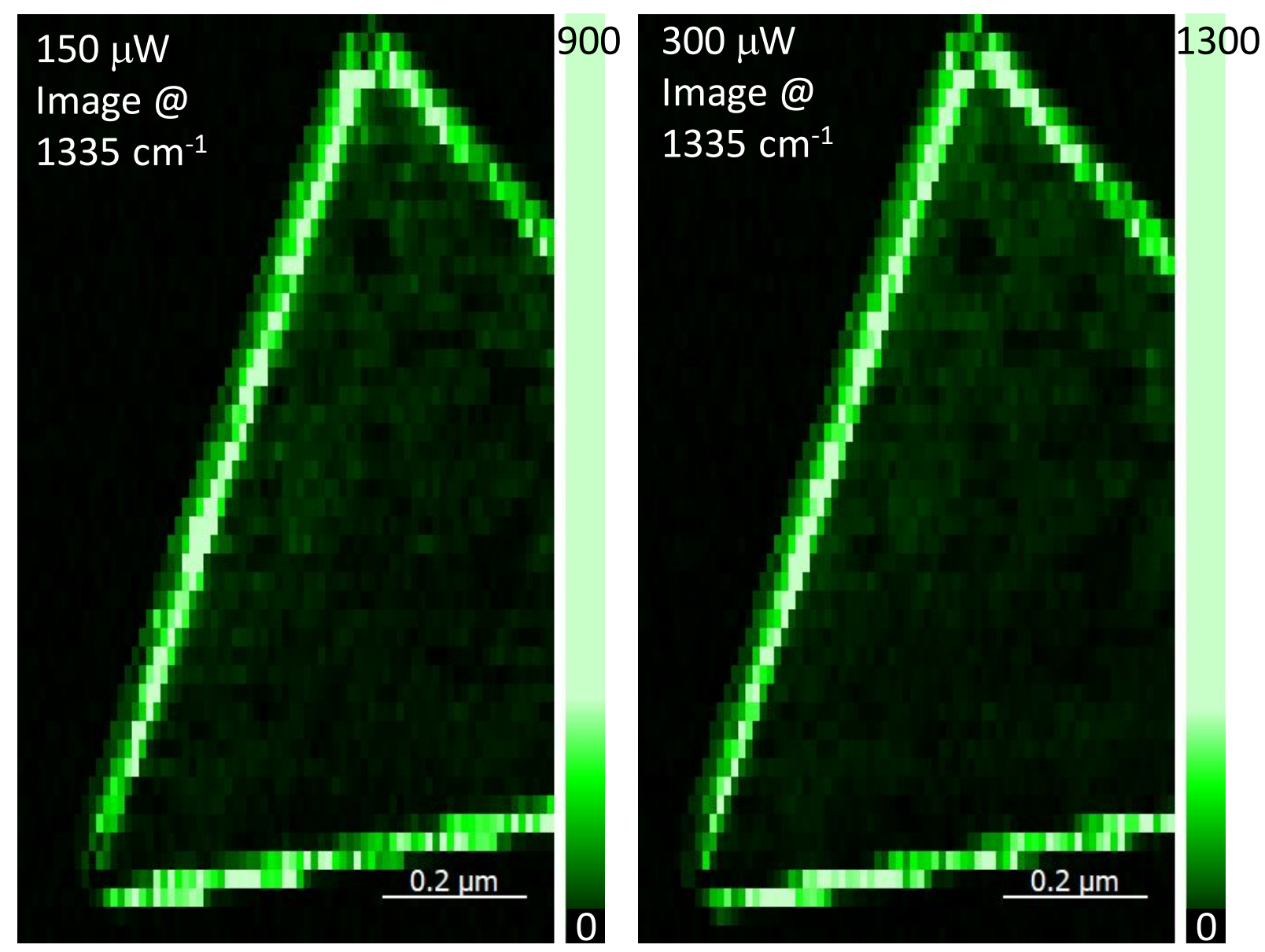

Figure S1. TERS images (at $1335 \mathrm{~cm}^{-1}$ ) recorded simultaneously with their analogues that are shown in Figures $3 \mathrm{~A}$ and $3 \mathrm{~B}$ of the main text. All parameters are given in the caption of Figure 1 in the main text. The only difference is the power of the incident laser (shown in the insets). 

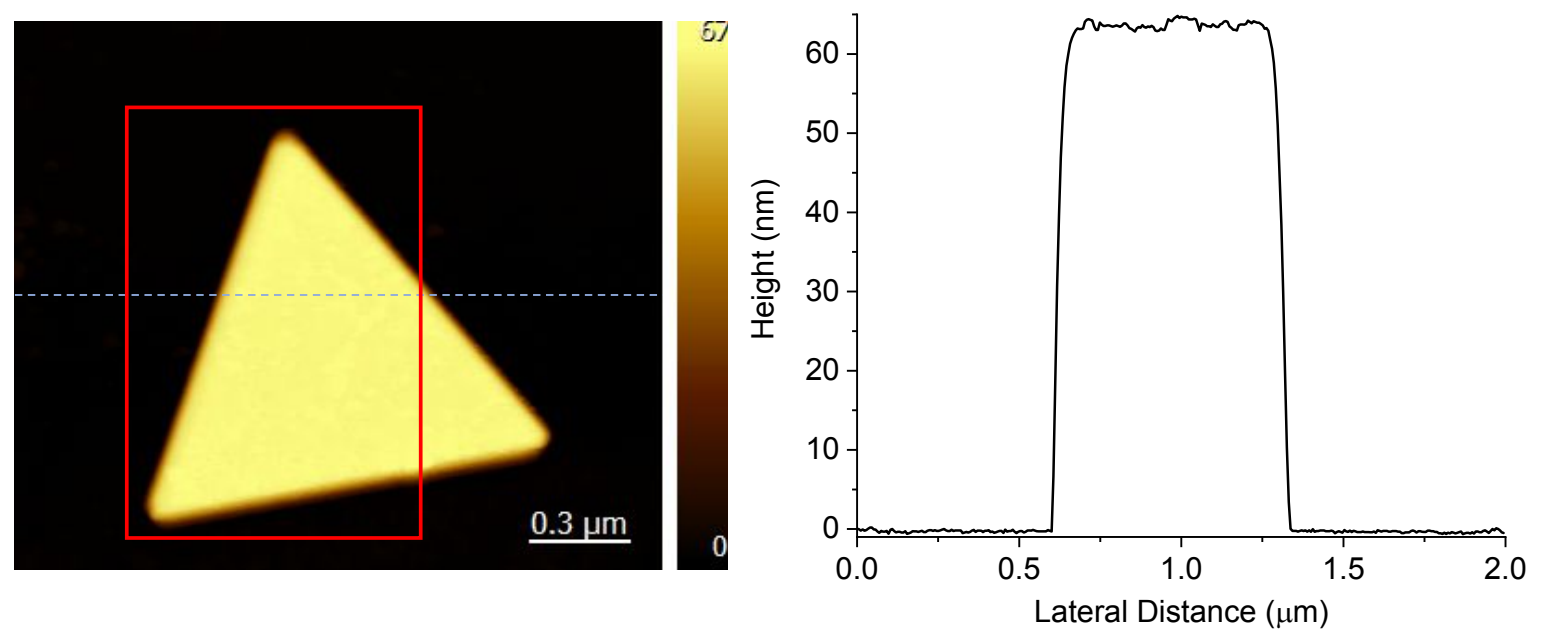

Figure S2. AFM characterization of the nanoplatelet analyzed via TERS in the main text. The highlighted red region in the left panel was visualized through TERS throughout this study. The dashed horizontal line indicates the position at which the cross-sectional cut (right panel) was taken. 

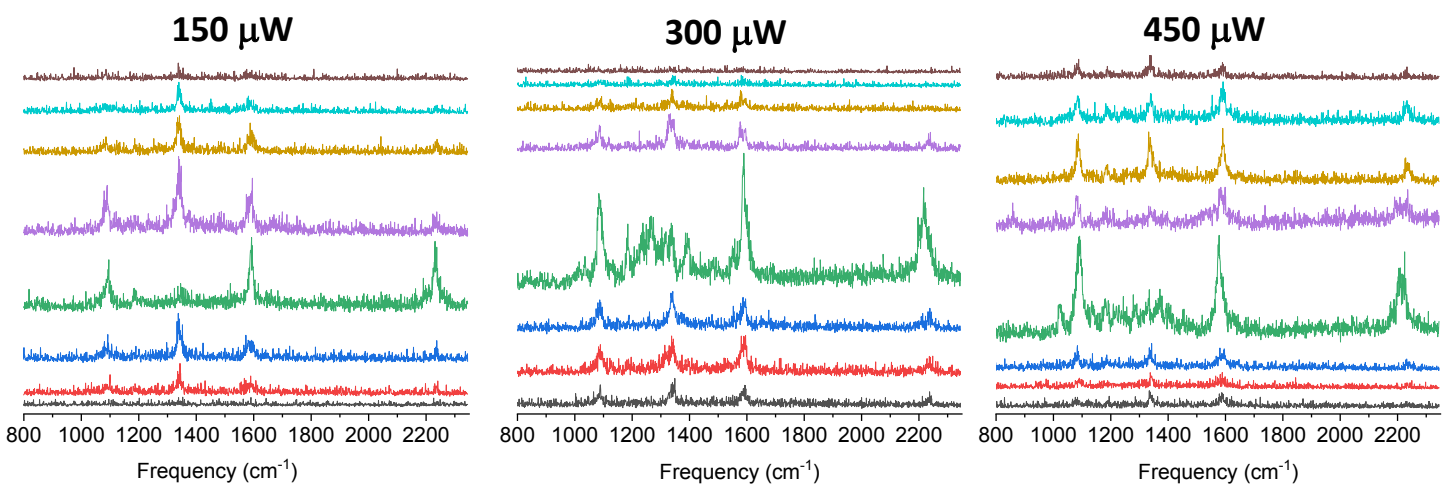

Figure S3. TERS cross-sectional line cuts taken at the positions indicated in Figures $3 \mathrm{~A}$ and $3 \mathrm{~B}$ of the main text. The right panel $(450 \mathrm{~mW})$ adds yet another data point that supports our claim of pixel-limited spatial resolution based on the dual analyte approach. The left and middle panel are duplicates of Figures $3 C$ and $3 D$ in the main text. 